\title{
GEO-Pot: Seichtes Geothermie Potenzial Österreichs. Überregionale, interdisziplinäre Potenzialstudie zur Erhebung und Darstellung des oberflächennahen geothermischen Anwendungspotenzials auf Grundlage eines regelmäßigen Bearbeitungsrasters
}

\begin{abstract}
Zusammenfassung: Im Rahmen der Studie GEO-Pot wurde ein flächendeckendes Rasterzellenmodell im Maßstab 1:500.000 erarbeitet, das aufgrund der Verknüpfung von geowissenschaftlichen Basisdaten, technischen Modellen und raumplanerischen Kennwerten eine generalisierte Prognose zum oberflächennahen, geothermischen Potenzial Österreichs liefert. Die Grundlage des Rasterzellenmodells bildet ein raumplanerisches Modell, welches potenzielle Nutzungs- und Ausschlussflächen eingrenzt. Den dadurch bekannten und soweit geothermisch nutzbaren Flächen wurde ein generalisiertes Untergrundmodell zugewiesen, das die Berechnung der potenziellen technischen Entzugsleistung mithilfe der Simulation auf Grundlage von Referenzgewinnungsanla-

of potential geothermal usage and areas to be excluded. The areas found that lent themselves to geothermal usage were associated with a generalised ground model, which allowed the potential technical extraction capacity to be calculated by means of a simulation on the basis of reference production plant (vertical collector, shallow collector). It was thus possible to determine the geothermal potential (technical potential, the potential to meet requirements) in combination with the demand situation, that is, the specific heating demand related to housing units. Building types were defined, using structural and site-specific building data, and these were associated with heating-demand data on the assumption of heating refurbishment being carried out.
\end{abstract} gen (Tiefensonde, Flachkollektor) ermöglicht hat. Die Bestimmung des geothermischen Potenzials (technisches Potenzial, Deckungspotenzial) konnte in Kombination mit der erhobenen Bedarfssituation, sprich der Identifizierung des ortsbezogenen Heizwärmebedarfs bezogen auf siedlungsstrukturelle Einheiten, durchgeführt werden. Hierzu wurden aufgrund bautechnischer und standortbezogener Gebäudedaten Gebäudetypen definiert, denen unter Annahme einer Sanierung Heizwärmebedarfswerte zugewiesen wurden

GEO-Pot: Shallow geothermal potential in Austria. Supraregional and interdisciplinary study to determine the shallow geothermal application potential on the basis of a regular grid cell model

Summary: A comprehensive grid cell model was prepared at a scale of 1:500.000, linking basic data in terms of the Earth sciences and regional planning as well as technical models, to permit a generalised forecast to be made on the near-surface geothermal potential in Austria. The basis of the grid cell model was a regional planning model delimiting areas

\section{Einleitung}

Bei der Potenzialerhebung erneuerbarer Energien wird die oberflächennahe Geothermie meist nur als Randthema behandelt, obwohl sie ein beachtliches Nutzungspotenzial aufweist. Dies begründet sich darin, dass die technische Umsetzbarkeit auf nationaler Ebene bei weitem schwieriger zu beurteilen ist als jene anderer Energieformen. Das Projekt GEO-Pot ist eine fundierte Grundlagenstudie unter Beteiligung verschiedener Wissenschaftsdisziplinen zur Evaluierung des oberflächennahen geothermischen Nutzungspotenzials auf Grundlage eines interdisziplinären Rasterzellenansatzes im Maßstab 1:500.000. Die Studie beschränkt sich hierbei auf die Gewinnungssysteme „Tiefensonde“ und „Flachkollektor“. Grundwasser-Wärmepumpen, Luft-Luft bzw. Luft-Wasser Wärmepumpen sowie unkonventioneller Sondensysteme (z. B. $\mathrm{CO}_{2}$-Sonden) mussten ausgeklammert werden. Auf Grundlage eines flächendeckenden Rasters mit einer Zellenlänge von 250 x 250 Meter wurde eine
Vorgehensweise erarbeitet, die es erstmals erlaubt, aufbereitete geowissenschaftliche Basisdaten mit technischen Modellen und raumplanerischen Kennwerten zu verknüpfen.

\section{Erhebung des räumlichen Dargebots}

Das Nutzungspotenzial oberflächennaher, wärmepumpenunterstützter geothermischer Anwendungen wird von den räumlichen Gegebenheiten beeinflusst. Im Gegensatz zur Nutzung der „tiefen Geothermie“" z. B. in Form der Gewinnung natürlicher Thermalwässer, spielen die thermischen Leitungs- und Speichereigenschaften sowie das vorherrschende Temperaturniveau im Untergrund nur eine verminderte Rolle gegenüber den jeweils zur Verfügung stehenden Nutzungsflächen. Es sei an dieser Stelle betont, dass das Wirkungsprinzip der oberflächennahen Geothermie im Wesentlichen auf den thermischen Speichereigenschaften des Untergrunds (Akkumulatorwirkung) basiert, wobei die künstlich erzwungenen Wärmeströme zwischen dem umgebenden Untergrund und dem Absorber den natürlichen Wärmefluss (terrestrische Wärmestromdichte) etwa um den Faktor 10.000 übertreffen. In Anbetracht der zur Verfügung stehenden Projektmittel wurde ein pragmatischer Ansatz erarbeitet, der es erlaubt aus verfügbaren Eingangsdaten ein generalisiertes Raummodell unter Berücksichtigung des gewählten Betrachtungsmaßstabes von 1:500.000 zu definieren. Dieses Raummodell setzt sich primär aus einem geowissenschaftlichen und einem raumplanerischen Modell zusammen.

\subsection{Geowissenschaftliches Daten- modell}

Das geowissenschaftliche Ausgangsmodell dient dazu die geogenen Voraussetzungen der oberflächennahen geother- 
mischen Nutzung dem vorgegebenen Maßstab von 1:500.000 anzunähern und für eine Verarbeitung auf Rasterzellenebene (Zellengröße $250 \mathrm{~m}$ x $250 \mathrm{~m}$ ) aufzubereiten. Es setzt sich aus einer generalisierten Prognose des geologischen Aufbaus und dessen Charakterisierung mit technischen Kennzahlen sowie aus der Erstellung von Bodentemperaturprognosen zusammen.

\subsubsection{Geologie}

Eine wesentliche Herausforderung bestand hierbei in der Vereinfachung des drei - dimensionalen Untergrunds zu zweidimensionalen Projektionen. Dies führt vor allem im Bereich von Beckenund Talrändern zu gewissen Ungenauigkeiten, da sich dort die Tiefenlage der Beckenbasis kleinräumig stark ändern kann. Ausgangspunkt der geologischen Modellierung bildet die Hydrogeologische Karte Österreichs im Maßstab 1:500.000 (Schubert 2003). Die bestehende Karte wurde im Bereich außeralpiner und inneralpiner Becken mit anzunehmenden Änderungen des geologischen, respektive des lithologischen Aufbaus des Untergrunds mit zusätzlichen Homogenbereichen versehen. In weiterer Folge wurde jedem Homogenbereich ein geologisches Referenzprofil zugewiesen, welches den Tiefenbereich von 0 bis 150 Meter unter Geländeoberkante (GOK) beschreibt. Die hierfür notwendigen Eingangsinformationen wurden aus Bohrprofilen kompiliert, die in den Archiven der Geologischen Bundesanstalt aufliegen. Hierdurch konnte der Untergrund Österreichs durch insgesamt 41 geologische Referenzprofile abgebildet werden. Diese Referenzprofile, die als virtuelle Bohrprofile zu verstehen sind, beinhalten petrografische Beschreibungen der beteiligten geologischen Schichten, die sich aus Gesteinstypen entsprechend der ÖNORM B4401 ergeben. Da in den meisten Schichten Übergänge verschiedene Gesteinstypen teilweise alternierend anzutreffen sind, wurden Mischmodelle einzelner Gesteinstypen durch gewichtete Mittelung gemäß den angenommenen volumetrischen Anteilen berechnet. Die erarbeiteten geologischen Referenzprofile bilden den Ausgangspunkt der petrophysikalischen und thermischen Prognosemodelle und stellen die Projektion des drei - dimensionalen Untergrunds auf ein zweidimensionales Bearbeitungsraster dar.

TABELLE 1

Übersichtstabelle der thermischen Gesteinseigenschaften der benutzten individuellen Gesteinstypen (Typisierung nach ÖNORM B4401).

\begin{tabular}{|c|c|c|c|c|}
\hline \multirow[t]{2}{*}{ ID $^{1}$} & \multirow[t]{2}{*}{ Bezeichnung' } & \multicolumn{2}{|c|}{$\begin{array}{l}\text { Effektive thermische Eigenschaften } \\
\text { unter Berücksichtigung von Porenanteil } \\
\text { und Porenfüllung (Rechenwert) }\end{array}$} & \multirow[t]{2}{*}{ Quelle } \\
\hline & & $\begin{array}{l}\text { Wärmeleitfähigkeit } \\
\text { W/(m.K) }\end{array}$ & $\begin{array}{l}\text { Spez. Wärmekapazität } \\
\text { J/(kg.K) }\end{array}$ & \\
\hline k. A. & Humus & 0,45 & 780 & 1 \\
\hline 4 & Kies (wassergesättigt) & 1,80 & 889 & 1 \\
\hline 4 & Kies (trocken) & 0,40 & 761 & 1,2 \\
\hline 9 & Sand & 2,40 & 960 & $1,2,3$ \\
\hline 13 & Schluff (trocken) & 0,45 & 780 & 1,2 \\
\hline 13 & $\begin{array}{l}\text { Schluff (wassergesät- } \\
\text { tigt) }\end{array}$ & 1,80 & 1923 & 1,2 \\
\hline 14 & Ton & 1,40 & 1629 & 1 \\
\hline 18 & Löß & 0,58 & 1010 & -- \\
\hline 20 & Lohle & 0,40 & 1400 & 2 \\
\hline 24 & Konglomerat & 2,80 & 900 & 1,2 \\
\hline 26 & Sandstein & 2,46 & 737 & 1,2 \\
\hline 27 & $\begin{array}{l}\text { Sandstein (schwach } \\
\text { verfestigt) }\end{array}$ & 1,20 & 972 & $1,2,3$ \\
\hline 31 & Kalkstein & 2,67 & 902 & 1,2 \\
\hline 33 & Mergelstein & 2,15 & 868 & 1,2 \\
\hline 35 & Dolomit & 3,22 & 1088 & $1,2,3$ \\
\hline 62 & Granit & 3,40 & 1110 & 2,3 \\
\hline 1001 & Tonmergel & 2,00 & 866 & -- \\
\hline 1002 & Mergel & 2,10 & 860 & 1 \\
\hline 66 & Trachyt, Andesit & 2,80 & 900 & 2 \\
\hline 54 & Gneis & 2,70 & 819 & 1,2 \\
\hline 66 & Amphibolit & 2,90 & 900 & 2 \\
\hline 53 & Glommerschiefer & 3,75 & 800 & 1,2 \\
\hline 48 & Quarzphyllit & 3,62 & 818 & 2 \\
\hline 47 & Phyllit & 2,43 & 900 & 1,2 \\
\hline 57 & Quarzit & 5,87 & 796 & 1,2 \\
\hline 30 & Tonschiefer & 2,13 & 921 & 1,2 \\
\hline 41 & Anhydrit & 4,10 & 714 & 2 \\
\hline 29 & Tonstein & 1,96 & 885 & $1,2,3$ \\
\hline
\end{tabular}

Bezeichnung gemäß ÖNORM B4401, EN ISO 22475-1: Geotechnische Erkundung und Untersuchung.

1001 Dreistellige Nummerierungen repräsentieren Gesteinsmischtypen, die in der ÖNORM B4401 nicht enthalten sind.

Quellenangaben:

Verein Deutscher Ingenieure, VDI Richtlinie 4640, Blatt 1: Thermische Nutzung des Untergrundes, Düsseldorf 2008

Schön J, Petrophysik - Physikalische Eigenschaften von Gesteinen und Mineralien, Akademie Verlag Berlin 1983

3 Kutasov I. M., Applied geothermics for petroleum engineers, Developments in Petroleum Science Vol. 48, Elsevier 1999

Daten wurden geschätzt bzw. Analogieschluss zu vergleichbaren Gesteinstypen

Die Auswahl von Rechenwerten erfolgte durch Mittelung bzw. Abgleich vorhandener Literaturdaten, die sich auf teilweise unterschiedliche Wassersättigungsniveaus und Porositäten beziehen.

In einem ersten Bearbeitungsschritt wurden die in den zuvor beschriebenen Referenzprofilen ausgewiesenen Gesteinstypen gemäß ÖNORM B4401 mit Literaturkenndaten zu den Parametern (a) bis (c) versehen (siehe auch Tab. 1). Hierauf basierend wurden anschließend Mischmodelle für die effektiven thermischen Schichteigenschaften auf Grundlage der volumetrischen Anteile der Einzelgesteinstypen wie folgt nach Schön (1983) gemittelt: 


\begin{tabular}{|c|c|c|c|c|c|}
\hline Bauteil & EFH 1-2 WE [m²] & MFH 4WE $\left[\mathrm{m}^{2}\right]$ & MFH 8WE [m²] & MFH 12WE [m²] & MFH 32WE $\left[\mathrm{m}^{2}\right]$ \\
\hline Dach & 108 & 216 & 360 & 320 & 720 \\
\hline Aussenwände & 163 & 341 & 508 & 962 & 1834 \\
\hline Kellerdecke & 99 & 216 & 360 & 320 & 720 \\
\hline Fenster & 41 & 79 & 120 & 228 & 444 \\
\hline Bruttogeschoßfläche & 198 & 432 & 720 & 1280 & 2880 \\
\hline
\end{tabular}

TABELLE 3

U-Werte und g-Werte der thermisch sanierten Wandaufbauten und Fenster für das Referenzgebäude der unterschiedlichen Bauperioden

\begin{tabular}{|c|c|c|c|c|c|c|}
\hline Bauperiode & $\mathrm{KD}\left[\mathrm{W} / \mathrm{m}^{2} \mathrm{~K}\right]$ & AW $\left[\mathrm{W} / \mathrm{m}^{2} \mathrm{~K}\right]$ & $\mathrm{OD}\left[\mathrm{W} / \mathrm{m}^{2} \mathrm{~K}\right]$ & $\mathrm{DG}\left[\mathrm{W} / \mathrm{m}^{2} \mathrm{~K}\right]$ & FE_U-Wert [W/m²K] & FE_g-Wert [-] \\
\hline bis 1945 & 0,380 & 0,540 & 0,710 & 0,160 & 1,40 & 0,60 \\
\hline 1946-1980 & 0,367 & 0,171 & 0,164 & 0,142 & 1,40 & 0,60 \\
\hline 1981-1990 & 0,289 & 0,155 & 0,184 & 0,166 & 1,40 & 0,60 \\
\hline 1991-2001 & 0,247 & 0,150 & 0,165 & 0,150 & 1,10 & 0,58 \\
\hline nach 2001 & 0,232 & 0,141 & 0,140 & 0,131 & 1,10 & 0,58 \\
\hline
\end{tabular}

- Effektive Wärmeleitfähigkeit (geometrische Mittelung)

$$
\lambda_{\text {eff }}=\prod_{i}\left(\lambda_{i}^{C_{i}}\right)
$$

- Effektive spezifische Wärmekapazität (arithmetische Mittelung)

$$
\mathrm{c}_{\mathrm{p}, \mathrm{eff}}=\sum_{\mathrm{i}}\left(\mathrm{c}_{\mathrm{p}, \mathrm{i}} \cdot \mathrm{c}_{\mathrm{i}}\right)
$$

$c_{i} \ldots$ volumetrische Anteile

Da die Schwankungsbreite der effektiven Gesteinsdichte (Raumgewicht) im betrachteten Tiefenbereich der Studie GEO-Pot nicht sehr ausgeprägt ist wurde auf eine explizite Modellierung der effektiven Gesteinsdichte verzichtet. Aus Übersichtsgründen wurde auf eine explizite Modellierung der effektiven Gesteinsparameter unter Berücksichtigung von Porenanteilen und Porenfluidmodellen (Wasser verschiedener Mineralisation, Luft) verzichtet. Die zu Grunde liegenden gesteinsspezifischen Kennwerte berücksichtigen deshalb bereits implizite Annahmen der Porenanteile in den jeweiligen Gesteinstypen bei Abschätzung der Wassersättigung. Es sei jedoch abschließend darauf hingewiesen, dass es sich hierbei um synthetische thermische Gesteinskennwerte handelt, die aus Literaturdaten berechnet worden sind. Eine Validierung dieser Modelle anhand von in-situ Messungen ist im Rahmen der Studie GEO-Pot leider nicht möglich.

\subsubsection{Prognose der Bodentempera-} turen

Zur Bestimmung der technischen Entzugsleistung geothermischer Anwendungen ist neben der Prognose der thermischen Leitungs- und Speichereigenschaften des Untergrunds die Vorhersage der Bodentemperatur in verschiedenen Tiefen von großer Bedeutung. Im Rahmen der Studie
GEO-Pot wurde das folgende, vereinfachte Vorhersagemodell der Bodentemperatur in Abhängigkeit der Seehöhe und des geologischen Aufbaus des Untergrunds (n-Schicht Fall) verwendet:

$$
\mathrm{T}(\mathrm{h}, \mathrm{z})=\mathrm{T}_{0}(\mathrm{~h})+\sum_{\mathrm{i}=1}^{\mathrm{n}}\left(\frac{\mathrm{z}_{\mathrm{i}}-\mathrm{z}_{\mathrm{i}-1}}{\lambda_{\text {eff,i }}}\right) \cdot \mathrm{q}
$$

Unter Vernachlässigung der periodisch schwankenden Amplitude der Bodentem-

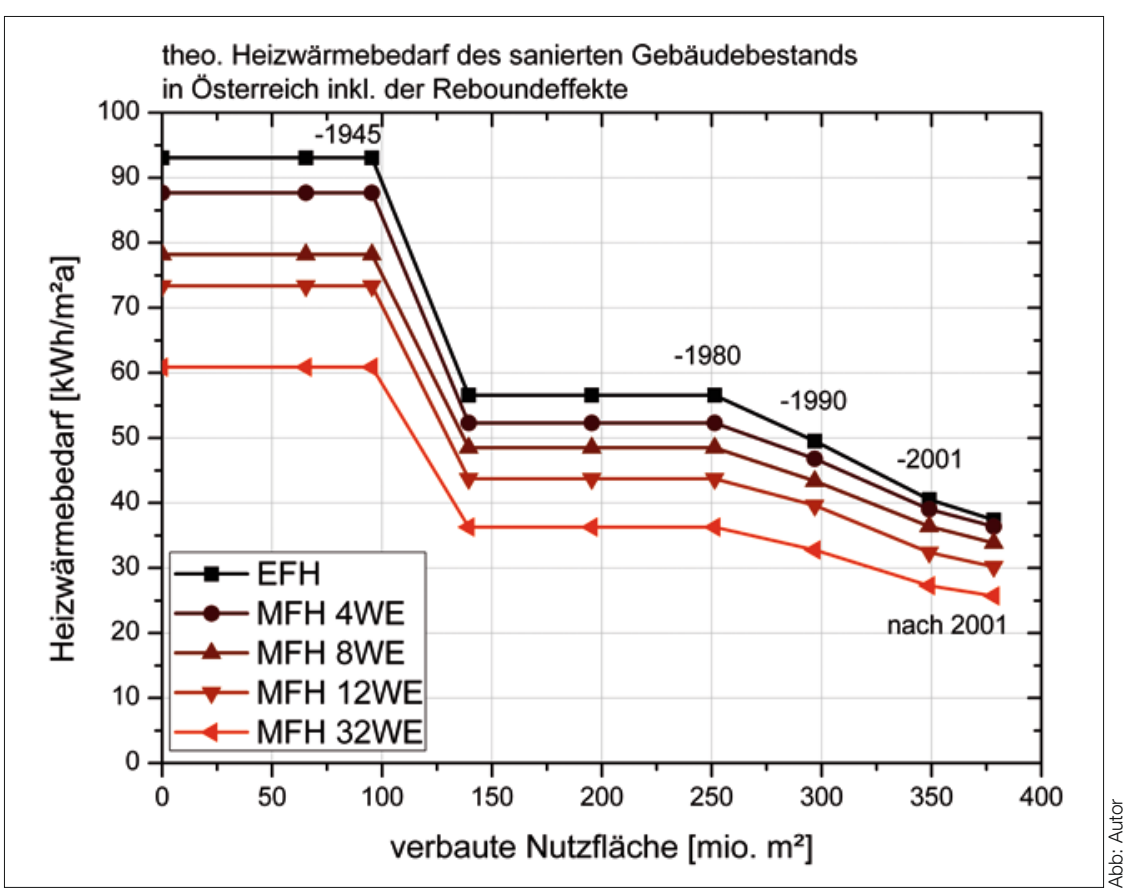

Abb. 1: Theoretischer Heizwärmebedarf im thermisch sanierten österreichischen Wohnbau unter Berücksichtigung der Reboundeffekte. 

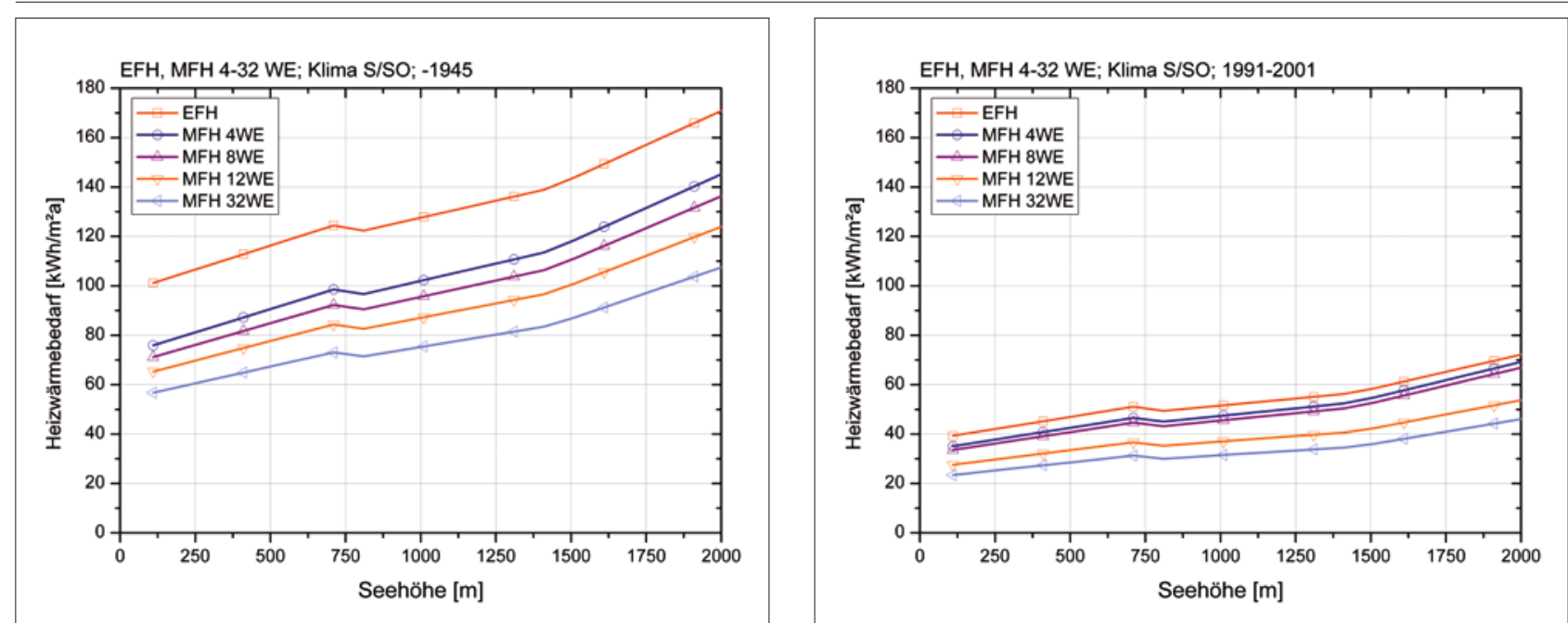

Abb. 2: Theoretischer Heizwärmebedarf des thermisch sanierten österreichischen Wohnbaus für unterschiedliche Bauperioden in der Klimazone S/SO in Abhängigkeit der Seehöhe.

peratur in dem durch den Jahresgang der Lufttemperatur beeinflussten Tiefenbereich von 0 bis max. 30 Meter unter Gelände wird die Bodentemperatur an der Basis der Schicht $i$ in der Tiefe $z_{i}$ rekursiv unter Vorgabe eines konstanten terrestrischen Wärmeflusses $\left(\mathrm{q}=70 \mathrm{~mW} / \mathrm{m}^{2}\right)$ berechnet. Die Oberflächentemperatur $T_{0}$ wurde in Abhängigkeit von der Seehöhe $h$ empirisch aus Beobachtungsdaten der Zentralanstalt für Meteorologie und Geodynamik (ZAMG) bestimmt. Hierfür wurden Temperaturmessdaten in $20 \mathrm{~cm}$ und $50 \mathrm{~cm}$ Tiefe aus 98 Stationen ausgewertet, die im Zeitraum 1997 bis 2007 erhoben worden sind. Hieraus konnte folgende, auf den Messdaten in $20 \mathrm{~cm}$ Tiefe basierende lineare Interpolationsfunktion der Oberflächentemperatur abgeleitet werden:

$$
\mathrm{T}_{0}(\mathrm{~h})=12.52-4.1 \cdot 10^{-3} \cdot \mathrm{h}
$$

Die Interpolationsfunktion besitzt einen Anpassungsgrad $\left(\mathrm{R}^{2}\right)$ von $93 \%$ und genügt somit der gewünschten Präzision der Studie GEO-Pot. Es hat sich zudem gezeigt, dass eine Gliederung der zur Verfügung stehenden Rohdaten in verschiedene Klimazonen Österreichs zu keiner signifikanten Verbesserung des Anpassungsgrads führte. Streuungen der Messwerte um die Regressionsgerade sind in erster Linie auf lokale Einflüsse, wie Hanglage, Bewuchs oder Grundwassereinflüsse zurückzuführen.

\subsection{Raumplanerisches Ausgangs- modell}

Die Identifizierung von potenziellen Ausschlussflächen erfolgte gemäß wasser- wirtschaftlicher und raumplanerischer Überlegungen. Wasserwirtschaftliche Ausschlussflächen stellen Grundwasserentnahmestellen sowie Grundwasserschutzgebiete dar. Raumplanerische Ausschlussflächen stellen neben hoch verdichteten Siedlungsräumen (z.B. urbane Zentren) jene als dauerhaft unbewohnbar anzusehende Flächen dar: Felswände, hochalpine Bereiche (Permafrost), Wasser- und Verkehrsflächen.

Um den Freiflächenanteil der besiedelten Zellen bestimmen zu können, wurde auf einen europaweit verfügbaren, satellitengestützten Datensatz zurückgegriffen, der in einem $100 \mathrm{~m}$-Raster die versiegelten Flächen in den jeweiligen Zellen anteilsmäßig erfasst. Diese $100 \mathrm{~m}$-Raster wurden für das vorliegende Ausgangsmodell in 250 m x 250 m Raster umgerechnet. Für jene Zellen, in denen zwar Gebäude vorhanden sind, jedoch keine Werte für die Versiegelung aus den Satellitendaten existieren (z. B. durch Datenfehler, Wolkenbedeckung während der Erfassung etc.) wurde die Versiegelungswerte, und hieraus die Freiflächenanteile über ein geeignetes Regressionsmodell aus den Werten der benachbarten statistischen Merkmale (wie Gebäude, Wohngebäude, Betriebe etc.) geschätzt.

\section{Abbildung der Bedarfssituation}

Die Grundlage für die Abbildung des Wärmebedarfs ist der Gebäudebestand mit seinen Charakteristika Gebäudetyp (Nutzung), Qualität der Gebäudehülle (Baujahr) und Standort. Mit Hilfe dieser Kenndaten kann der Heizwärmebedarf der Gebäude modelliert werden.
In Österreich sind ca. $47 \%$ aller Wohnungen in Gebäuden mit 1 oder 2 Wohnungen (Ein- und Zweifamilienhäuser, „EFH“) untergebracht. Etwa gleich viele Wohnungen sind in Gebäuden mit drei oder mehr Wohnungen („MFH“) zu finden (ca. $50 \%$ ). Der Rest entfällt hauptsächlich auf Wohnungen in Nichtwohngebäuden.

\subsection{Bestimmung des Heizwärme- bedarfs (HWB; Nutzenergieebene)}

Um den österreichischen Gebäudebestand und darauf aufbauend den HWB abbilden zu können, wurden Referenzgebäude erstellt, bei denen folgende Merkmale variiert wurden:

- Gebäudetyp (5 Typen; fixe Bruttogeschoßfläche je Typ): Einfamilienhaus (EFH), Reihenhaus (MFH-4WE), kleines Mehrfamilienhaus (MFH8WE), mittleres Mehrfamilienhaus (MFH-12WE), großes Mehrfamilienhaus (MFH-32WE)

- Bauperiode (5 Altersklassen): vor 1945, 1946-80, 1981-90, 1991-2001, nach 2001

- Seehöhe (Reliefhöhe)

- Klimazone (7 Zonen in Österreich, nach ÖNORM B 8110-5)

Für jeden Gebäudetyp wurde eine typische Gebäudehülle definiert, welche für alle Bauperioden gleich angenommen wurde. Die Angaben zu den Geometrien (Hüllflächen und Bruttogeschoßflächen) sind in Tabelle $2 \mathrm{zu}$ finden.

Um den sinnvollen Einsatz oberflächennaher Geothermie zur Wärmebedarfsdeckung gewährleisten zu können, 
ist es notwendig, den Gebäudebestand thermisch zu sanieren. Dazu wurden die ursprünglichen $\mathrm{U}$-Werte ${ }^{1}$ und g-Werte ${ }^{2}$ (festgelegt in Anlehnung an die OIB Richtlinie 6 (2007) sowie in Absprache mit dem stv. Landesenergiebeauftragten des Landes Steiermark) durch zusätzliche Wärmedämmung an den Wandaufbauten und Fenstertausch verbessert. Die Werte nach thermischer Sanierung sind in Tabelle 3 dargestellt.

Mit den beschriebenen Annahmen wurde der HWB der Gebäude mittels des Energieausweises im Monatsbilanzverfahren am Standort des Referenzklimas berechnet. Das Referenzklima bietet die Möglichkeit, Gebäude an einem Vergleichsstandort hinsichtlich ihrer thermischen Qualität zu vergleichen. Zur Ermittlung des tatsächlichen HWB ist die Kenntnis des Standortes mit seinen Merkmalen, der Seehöhe und der Zugehörigkeit zu einer der 7 Klimazonen in Österreich (s. ÖNORM 8110-5) notwendig.

In der Praxis hat sich gezeigt, dass der energetische Einspareffekt nach thermischer Sanierung zumeist unter den Erwartungen liegt. Unter bestimmten Randbedingungen kommt es zu gar keinen Einsparungen, in extremen Fällen sind sogar Verbrauchsanstiege zu beobachten. Dieser sogenannte Reboundeffekt bei thermischer Sanierung wird hier auf Basis der Arbeit von Biermayer et al. (2005) berücksichtigt. Dadurch erhöht sich wiederum der HWB geringfügig, der zuvor durch Sanierung gesenkt worden war. Abbildung 1 zeigt den Verlauf des HWB des in die fünf Gebäudetypen eingeteilten österreichischen Gebäudebestands bei Referenzklima nach thermischer Sanierung unter Berücksichtigung der Rebound-Effekte.

Die zuvor erwähnte Standortabhängigkeit des HWB ist in Abbildung 2 für die Klimazone S/SO und die Bauperiode „bis 1945“ und „1991 bis 2001“ exemplarisch dargestellt.

Die vorliegenden Gebäudekategorien (Bauperiode und Gebäudetyp) und deren höhen- und standortabhängiger Heizwärmebedarf können somit durch raumplanerische Methoden auf die besiedelten Gebiete aggregiert werden.

\footnotetext{
Der Wärmedurchgangskoeffizient U (U-Wert, früher $\mathrm{k}$-Wert) ist ein $\mathrm{Maß}$ für den Wärmestromdurchgang durch eine ein- oder mehrlagige Materialschicht (Wandaufbau, Glasscheibe), wenn auf beiden Seiten verschiedene Temperaturen anliegen.

2 Der Energiedurchlassgrad (auch g-Wert) ist ein $\mathrm{Ma}$ für die Durchlässigkeit von transparenten
} Bauteilen für den Energietransport.

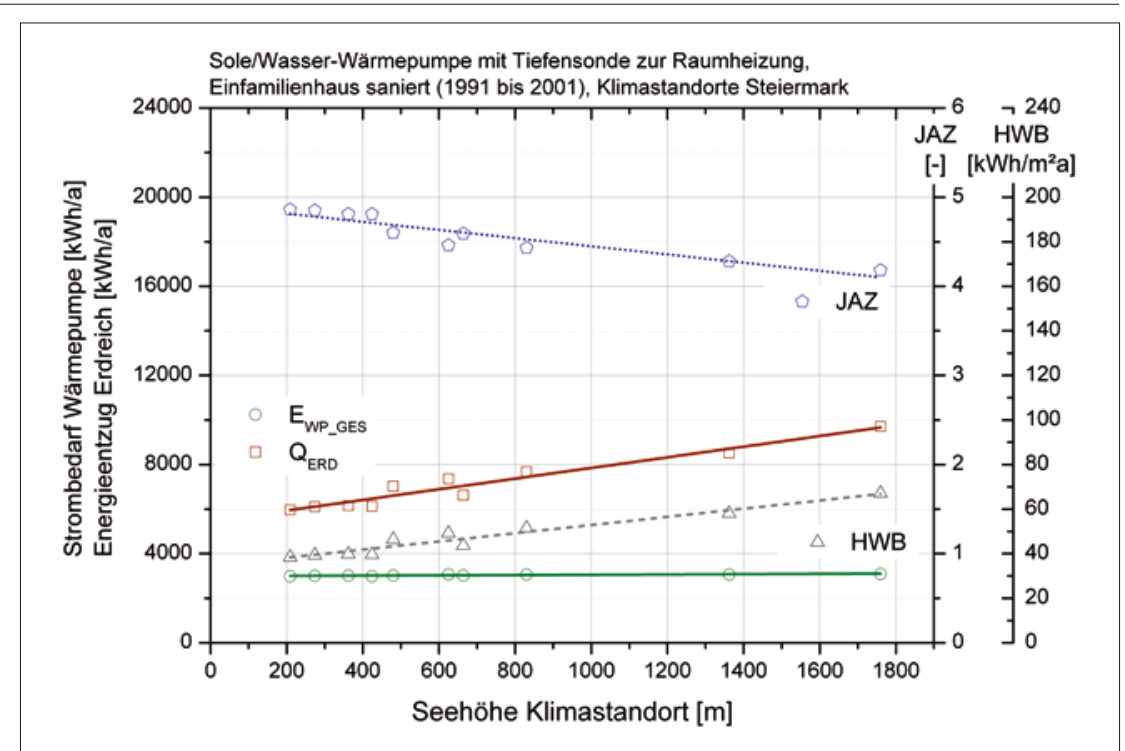

Abb. 3: Ergebnisse aus der thermischen Gebäude und Anlagensimulation (Polysun 5.2) für ein EFH mit Sole/Wasser-Tiefensonde und reiner Raumheizung.

\subsection{Heizenergiebedarf (HEB) und dem Erdreich mittels Wärmepumpen- technologie entzogene Energie}

Der Heizenergiebedarf auf Endenergieebene beinhaltet im Gegensatz zum Heizwärmebedarf, der auf Nutzenergieebene definiert ist, auch alle Verluste der Haustechnik. Um den notwendigen Heizenergiebedarf (HEB) der Gebäudetypen und jene Energiemenge, die dafür dem Erdreich $\left(\mathrm{Q}_{\mathrm{ERD}}\right)$ entzogen werden muss $\mathrm{zu}$ bestimmen, ist es notwendig, das gesamte Heizungssystem (Wärmequelle Erdreich über die Wärmepumpe bis hin zur Wärmesenke) mittels einer thermischen Gebäude- und Anlagensimulation abzubilden. Dazu wurde das Programm Polysun 5.2 (Marti 2009) verwendet. Die Dimensionierung der Systemkomponenten (Tiefensonde, Wärmepumpe) erfolgte in Anlehnung an die VDI Richtlinie 4640 (2001).

Aus der Simulation des Gesamtsystems an unterschiedlichen Standorten kann nun der Zusammenhang zwischen dem Heizwärmebedarf und dem Heizenergiebedarf $\left(\rightarrow \mathrm{f}_{\mathrm{HEB}}\right)$ sowie die Beziehung zwischen dem Heizwärmebedarf und dem Energieentzug aus dem Erdreich $\left(\rightarrow \mathrm{f}_{\mathrm{ERD}}\right)$ ermittelt werden.

In Abbildung 3 ist für eine Sole/Wasser Wärmepumpe mit Tiefensonde zur ausschließlichen Raumheizung für das Referenzgebäude EFH (1991-2001) der Verlauf von HWB, HEB, der Jahresarbeitszahl (JAZ; inkl. Pumpenstrom Solekreis) und dem Energieentzug aus dem Erdreich $\left(\mathrm{Q}_{\mathrm{ERD}}\right)$ über der Seehöhe an einigen Orten in der
Steiermark (Klimazone ZA bzw. S/SO) exemplarisch dargestellt.

Darin ist ersichtlich, dass der HWB mit der Seehöhe zunimmt. Die Jahresarbeitszahl (JAZ) sinkt mit zunehmender Seehöhe und der Entzug aus dem Erdreich $\left(\mathrm{Q}_{\mathrm{ERD}}\right)$ steigt annähernd parallel zum HWB.

Ausgehend vom standortabhängigen Heizwärmebedarf (HWB) kann somit über die Faktoren $\mathrm{f}_{\text {HEB }}$ und $\mathrm{f}_{\text {ERD }}$ der Heizenergiebedarf (HEB) und die notwendige Entzugsenergie aus dem Erdreich $\left(\mathrm{Q}_{\mathrm{ERD}}\right)$ ermittelt werden, die dann in die nachfolgende Potentialanalyse eingehen.

\subsection{Identifizierung des Gebäude-} bestands

Angesichts der vergleichsweise kurzen Versorgungsreichweite der oberflächennahen geothermischen Anlagen kommt der räumlichen Verteilung der Siedlungsstruktur bzw. Wohnnutzung besondere Bedeutung zu. Die Typisierung und räumliche Zuordnung siedlungsstruktureller Einheiten ist daher im Hinblick auf den Energiebedarf für Raumheizung ein wesentlicher Schritt für die Modellierung der räumlich disaggregierten Bevölkerungs-, Haushalts- und Wohnungssituation. Die Herausforderung bzw. Schwierigkeit liegt dabei darin, in der räumlich differenzierten Modellierung des Heizenergiebedarfs durch die Verschneidung von Raster- und Gemeindedaten die seitens der Gebäudetechnik spezifizierten Gebäudetypen zuzuordnen. Dazu werden die für jede Gemeinde in der Gebäude- und Wohnungszählung 2001 der amtlichen Statistik(Statistik Austria 2008) erfassten Daten 


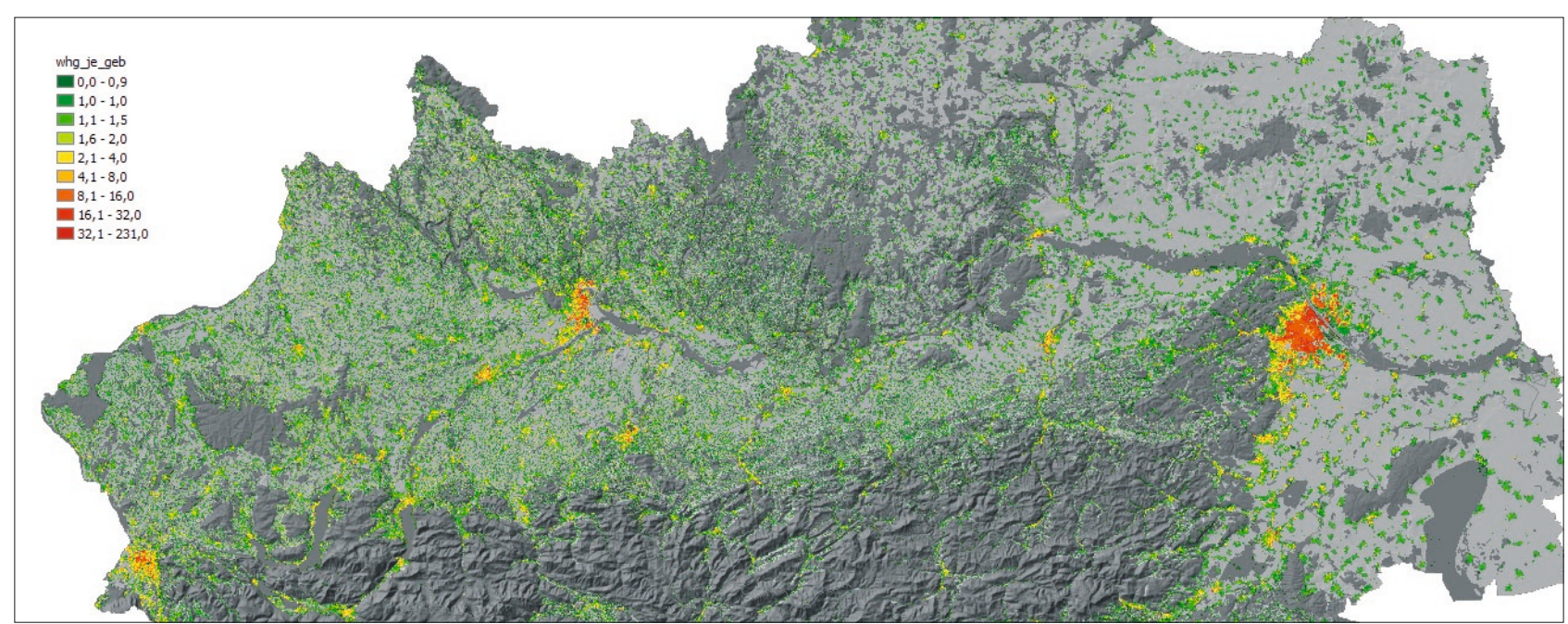

Abb. 4: Durchschnittliche Anzahl an Wohnungen je Gebäude, dargestellt in $250 \mathrm{~m}$ Raster.

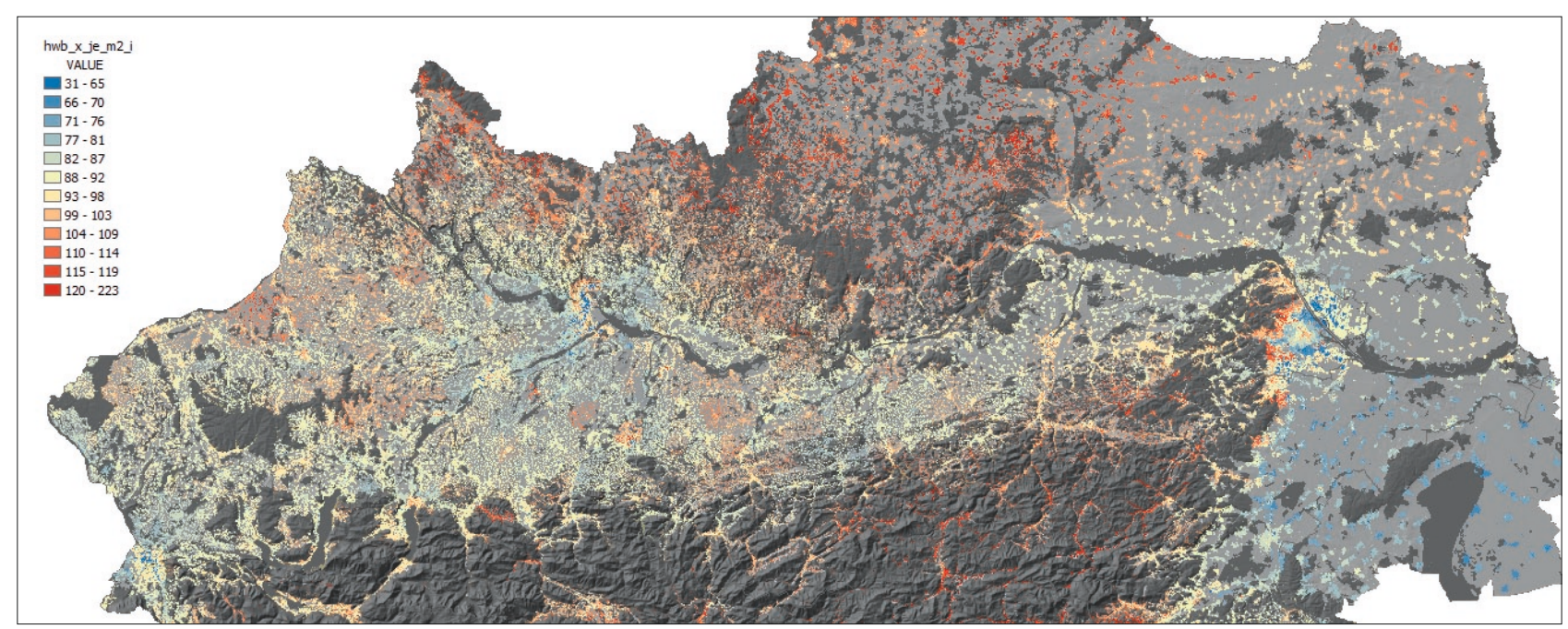

Abb. 5: Ausschnitt aus dem modellierten Heizwärmebedarf (HWB) je m² und Jahr (250m Raster).

zum Baualter der Gebäude, der Wohnungsgröße sowie der Anzahl Wohnungen je Gebäude mit den auf der kleinräumig disaggregierten Rasterebene (250m x $250 \mathrm{~m})$ verfügbaren Daten, die durchwegs als Berechnungsbasis sowohl auf Dargebotsals auch auf Nachfrageseite dienen, verschnitten. Dabei sind auf Rasterebene die Anzahl Gebäude (Wohngebäude, NichtWohngebäude), die Anzahl Haushalte, die Anzahl von Hauptwohnsitzen und Nebenwohnsitzen sowie die Anzahl der Wohnungen von entscheidender Bedeutung, um die räumliche Typisierung des Heizwärmebedarfs vornehmen zu können. Die kleinräumige Auflösung der zugrunde liegenden Daten wird in der nachfolgenden Karte beispielhaft veranschaulicht.

Die eingangs beschriebenen Gebäudetypen mit ihren jeweiligen typenpezifischen Energieverbrauchskennzahlen werden anhand der gemeindebezogenen
Daten unter Berücksichtigung der rasterbezogenen Informationen im Raum identifiziert, quantifiziert und zur Berechnung der Nachfrage (HWB) verwendet. Neben der Seehöhe und der Wohnungsgröße ist dabei das jeweilige Gebäudealter einer der wesentlichen Einflussparameter auf den Energiebedarf. Die Modellierung des HWB unter Berücksichtigung der tatsächlichen, kleinräumig differenzierten Siedlungsstruktur erfolgte unter Berücksichtigung der in Abschnitt 3.1 aufgelisteten Merkmale.

Der Gebäudetyp und die zugeordnete Bruttogeschoßfläche sowie die Bauperiode stehen in dieser räumlichen Differenzierung des $250 \mathrm{~m}$ Rasters aufgrund des Datenschutzes nicht zur Verfügung und müssen daher ausgehend von großräumiger aggregierten Daten sinnvoll modelliert werden. Der dominante Gebäudetyp je Raumeinheit bzw. Rasterzelle ergibt sich dabei aus der durchschnittlichen Anzahl der Wohnungen je Wohngebäude (und Rasterzelle). Die Verteilung der Gebäude nach Bauperioden muss dagegen gemäß der Verteilung in der jeweiligen Gemeinde auf die einzelnen besiedelten Rasterzellen umgelegt werden.

Pro Bauperiode und abhängig von den übrigen Parametern ergibt sich damit aus dem Modell der Heizwärmebedarf je $\mathrm{m}^{2}$ Bruttogeschoßfläche. Über die Zahl der Gebäude je Bauperiode und Raumeinheit sowie deren Bruttogeschoßfläche wird der Gesamtwärmebedarf für Raumheizung in weiterer Folge zellenweise aggregiert.

\section{Bestimmung des geotherm- ischen Nutzungspotenzials}

Das Nutzungspotenzial oberflächennaher geothermischer Anwendungen ergibt sich aus der Verknüpfung zweier Einzelpotenzi- 
ale: Dem technischen Potenzial (energetisches Dargebot) auf der Angebotsseite und dem Anwendungspotenzial auf der Bedarfsseite. Nicht jeder Wärmebedarf lässt sich durch oberflächennahe geothermische Anlagen decken. Einschränkend wirken sich hierbei das generell niedrige Temperaturniveau der Wärmequelle, der eng begrenzte technische Versorgungsradius sowie der Flächenbedarf der Erdwärmeanlage aus. Dies korrespondiert wiederum mit der Gebäudesubstanz (Alter und Sanierungsgrad des zu beheizenden Gebäudes).

\subsection{Bestimmung des technischen Potenzials}

Gemäß den Vorgaben der Studie GEO-Pot galt es einen zellenbezogenen Berechnungsansatz des technischen Potenzials $\left(Q_{\text {Gesamt }}\right) \mathrm{zu}$ definieren, der es erlaubt, alle relevanten Eingangsparameter (Geologie, Bodentemperatur, Siedlungsstruktur, Ausschlussflächen) in der zur Verfügung stehenden Auflösungsgenauigkeit zu berücksichtigen. Ausgangspunkt des gewählten Ansatzes bildet die zellenbezogene Bestimmung der technischen Entzugsleistung in Abhängigkeit vom Sondentyp (Geometrie, Betriebsweise), dem geologischen Aufbau des Untergrunds sowie der zu erwartenden Bodentemperatur. In einem ersten Bearbeitungsschritt wurde der spezifische Flächenbedarf der betrachteten Gewinnungssysteme $\left(A_{Q}\right)$ bestimmt. Dieser setzt sich aus der Querschnittsfläche der Sonde und dem thermischen Einflussbereich der Anlage zusammen, wobei letzterer in Zusammenhang mit dem geologischen Aufbau des Untergrunds gesetzt werden kann. Durch Verknüpfung einer flächenspezifischen Entzugsleistung $\left(Q_{A}\right)$ mit dem nutzbaren Flächenanteil einer spezifischen Zelle $\left(A_{Z}\right)$ lässt sich in weiterer Folge das technische Potenzial auf Zellenniveau ableiten. Formal lässt sich der gewählte Ansatz folgendermaßen beschreiben:

$$
\mathrm{Q}_{\text {Zelle }}=\underbrace{\frac{\mathrm{O}^{*}}{\mathrm{~A}_{\mathrm{Q}}}}_{\mathrm{Q}_{\mathrm{A}}} \cdot \mathrm{A}_{\mathrm{Z}}
$$

$\mathrm{Q}_{\text {Zelle }} \ldots$ Technische Entzugsleistung der spezifischen Zelle [kW] im Jahresmittel.

$\mathrm{Q}^{*}$... Techn. Entzugsleistung bei vorgegebenen Sondentyp und Betriebsweise $[\mathrm{kW}]$.

Durch Verknüpfung der technischen Entzugsleistung mit der Betriebsweise der Anlage (Jahresbetriebsstunden) lässt sich ohne erheblichen Berechnungsaufwand die technisch erzielbare Jahresarbeitsleistung [kWh/a] als kumulierter Gesamtwert für Österreich bestimmen.

\subsubsection{Abschätzung der technischen Entzugsleistung auf Grundlage der VDI Richtlinie 4640}

In der VDI Richtlinie 4640/Blatt 1 sind Richtwertbereiche der spezifischen thermischen Entzugsleistung [W je Meter Sondenlänge] unter Vorgabe folgender Gültigkeitsbedingungen angeführt (VDI 4640/ Blatt 1, S. 16):

- Die Jahresarbeitsleistung bei ausschließlichem Heizbetrieb unter Berücksichtigung von Warmwasseraufbereitung beträgt 1800 bis 2400 Stunden (Spezifische jährliche Entzugsarbeit $100-150[\mathrm{kWh} /(\mathrm{m} . \mathrm{a})])$.

- Die Länge der Wärmesonden beträgt 40 bis 100 Meter.

- Doppel-U-Rohr-Sonden mit einem Nenndurchmesser der Absorberleitungen von $20 \mathrm{~mm}, 25 \mathrm{~mm}$ oder $32 \mathrm{~mm}$.

Die VDI Richtlinie weist unter den zuvor genannten Gültigkeitsbedingungen Kennwertbereiche für gängige Gesteinstypen unter der Vorgabe von 1800 und 2400 Jahresbetriebsstunden auf und begründet sich auf empirischen Beobachtungen (Thermal Response Tests), die vornehmlich in Deutschland getätigt worden sind. Leider ermöglicht diese Kennwerttabelle nicht die spezifischen Entzugsleistungen in Abhängigkeit zur Standorthöhe zu setzen, die wiederum Einfluss auf die vorherrschenden Bodentemperaturen ausübt. Die Verknüpfung der Referenzprofile mit VDI-Kennwerten wurde schichtbezogen unter Zuhilfenahme gewichteter Mittelung (volumetrische Anteile) der beteiligten Gesteinstypen vollzogen. Durch Benutzung der VDI 4640 Richtwerte sollte (a) ein Erstüberblick über die zu erwartenden thermischen Entzugsleistungen und (b) die Möglichkeit einer Gegenüberstellung der häufig benutzten VDI 4640 Richtwerttabelle mit detaillierten numerischen Berechnungsmethoden ermöglicht werden.

Die Verknüpfung der geologischen Referenzprofile mit der VDI Richtlinie 4640 ergab, wie erwartet, dass die mittlere spezifische Entzugsleistung in Gebieten mit anstehendem Gebirge $(68.9 \pm 7.12 \mathrm{~W} / \mathrm{m}$ bei 1800 Jahresbetriebsstunden) um etwa $8 \%$ höher liegt als jene in Beckenregionen (63.2 $\pm 10.2 \mathrm{~W} / \mathrm{m})$. Innerhalb der einzelnen Profile treten je- doch starke Schwankungen auf. Es sei an dieser Stelle jedoch angemerkt, dass die VDI Richtlinie für bestimmte Gesteinsschichten (z. B. Kies) die Möglichkeit von fließendem Grundwasser berücksichtigt und hieraus stark erhöhte spezifische Entzugsleistungen resultieren.

Die Anwendung der VDI Richtlinie 4640 zur Abschätzung der Entzugsleistung von Flachkollektoren würde im Rahmen der Studie GEO-Pot zu trivialen Ergebnissen führen, da anhand der vorliegenden Bohrprofile keine Differenzierung der oberflächennahen Bodenschichten (Tiefe 0 bis 2 Meter unter GOK) durchgeführt werden konnte.

\subsubsection{Bestimmung der technischen Entzugsleistung auf Grundlage numerischer Simulationen}

Neben der Abschätzung der thermischen Entzugsleistung über Richtwerte der VDI 4640 wurde eine numerische Simulation auf Grundlage eines Finite-Volumen Algorithmus von Glück (2008) durchgeführt. Dies hat den Vorteil, die Abhängigkeit der thermischen Entzugsleistung von der Seehöhe in Form der Bodentemperatur berücksichtigen zu können. Die Geometrie der Tiefensonde sieht eine 90 Meter lange Duplexsonde mit einem Sondendurchmesser von $140 \mathrm{~mm}$ sowie einen Leitungsdurchmesser von $30 \mathrm{~mm}$ vor. Dieser Sondentyp wurde in Folge als Referenzsonde definiert (siehe auch Abb. 6). Die Simulation wurde unter Vorgabe der Eintrittstemperatur in die Sonde und der Durchflussrate für einen Zeitraum von 10 Betriebsjahren durchgeführt, wobeidieZeitschrittweite eine Stunde betrug. Die Eintrittstemperatur $\left(\theta_{\text {in }}\right)$ in die Sonde wurde aus Betriebskenndaten, erhoben durch das Austrian Institute of Technology (AIT) abgeleitet. In weiterer Folge wurde für alle geologischen Referenzprofile die spezifische thermische Entzugsleistung $[\mathrm{W} / \mathrm{m}]$ auf Grundlage der zuvor beschriebenen Referenzsonde für zwei unterschiedliche Seehöhen (400 m ü.A., 1000 m ü.A.) berechnet.

Da sich die thermische Entzugsleistung proportional zur Reliefhöhe verhält, kann in weiterer Folge bei der Bestimmung der Entzugsleistung für beliebige Seehöhen zwischen diesen beiden Stützstellen inter- bzw. extrapoliert werden. Die durchgeführten Simulationen ergaben folgende profilspezifischen Entzugsleistungen für eine Seehöhe von $400 \mathrm{~m}$ ü. A.

- Beckenregionen $55.9 \pm 2.4 \mathrm{~W} / \mathrm{m}$.

- Regionen mit anstehendem Festgestein: $57.9 \pm 2.4 \mathrm{~W} / \mathrm{m}$. 


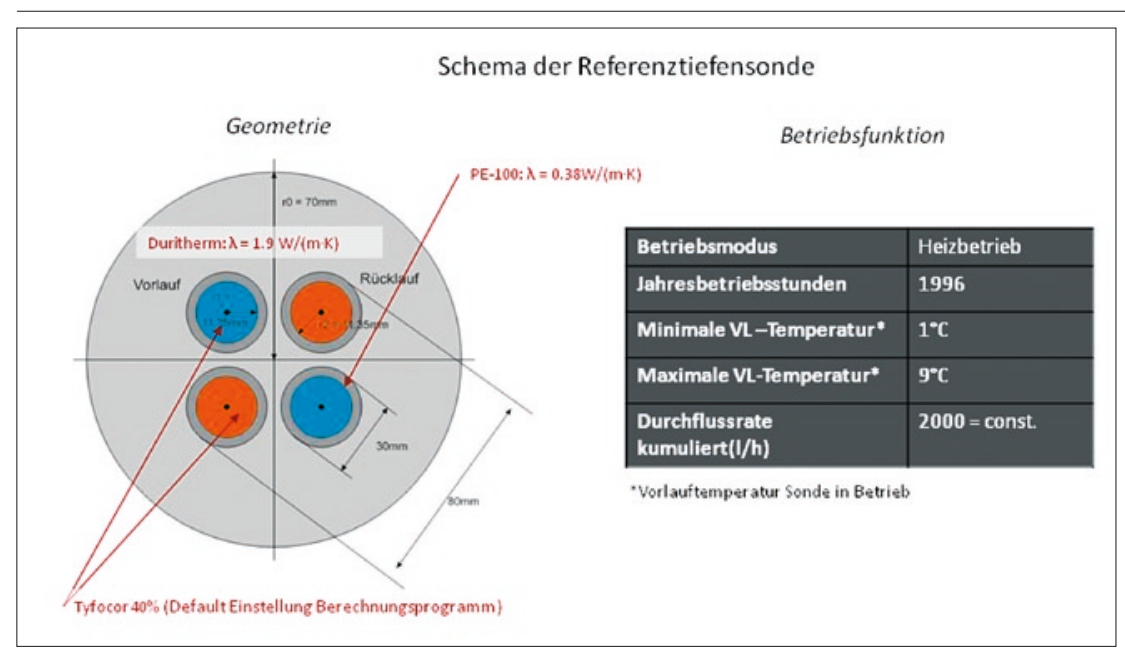

Abb. 6: Schema der Referenztiefensonde.

Im Vergleich zu den Ergebnissen der Erstabschätzung mittels VDI Richtlinie 4640 zeigen sich hier mit der Berechnung nach B. Glück (2008) wesentlich geringere Schwankungsbreiten der thermischen Entzugsleistung in Abhängigkeit vom geologischen Aufbau des Untergrunds. Als Begründung hierfür kann die Einschränkung, dass der Einfluss von fließendem Grundwasser in dieser Simulation ausgeblendet wird, genannt werden. Gemäß den vorliegenden Ergebnissen nimmt die thermische Entzugsleistung auf einer Seehöhe von $1000 \mathrm{~m}$. ü. A. um etwa $25 \%$ $(42.7 \pm 2.2 \mathrm{~W} / \mathrm{m})$ gegenüber den gemittelten Entzugsleistungen auf einer Höhe von $400 \mathrm{~m}$ ü. A. ab. Als primäre Ursache hierfür kann die Reduktion der Bodentemperatur infolge geringerer Lufttemperaturen genannt werden.

Glück (2009) liefert auch ein Simulationstool zur Prognose der Entzugsleistungen von Flachkollektoren. Da im Rahmen der Studie GEO-Pot keine Bodentypisierungskarten zur Verortung der thermischen Bodeneigenschaften zur Verfügung stehen, erfolgte eine vereinfachte Abschätzung der spezifischen thermischen Entzugsleistung von Flachkollektoren $\left[\mathrm{W} / \mathrm{m}^{2}\right]$ in Abhängigkeit der Bodentemperatur.

\subsection{Bestimmung des technischen Deckungsvermögens}

Wie bereits eingangs dieses Abschnitts erwähnt, ergibt sich das geothermale Deckungsvermögen aus dem Quotienten des technischen Potenzials und des Heizbedarfs einer spezifischen Zelle. Hierbei wird folgender Berechnungsansatz verfolgt:

Nach Abschluss der Wärmebedarfsermittlung in Abhängigkeit von den Gebäudetypen (Bautyp, Baualter) sowie der An- zahl an Gebäuden pro Zelle liegen zellenbezogene Summenwerte des Heizenergiebedarfs (HEB) vor $[\mathrm{kWh} / \mathrm{a}]$. Abhängigkeit vom Gebäudetyp, der Seehöhe und von den klimatischen Zonen der Zelle ergeben sich individuelle Jahresarbeitsstunden der Erdwärmeanlage. Die Bestimmung des geothermischen Deckungsgrads basiert auf der geforderten Bodenentzugsleistung $\left(\mathrm{Q}_{\mathrm{ERD}}\right)$, welche auf den HEB der auf der Zelle erwartenden Gebäudetypen abgestimmt worden ist, und der tatsächlich verfügbaren, längenspezifischen Entzugsarbeit pro Zelle $\left(Q_{\mathrm{L} \text {, zelle }}^{*}\right)$. Hierbei wird über den statistisch ermittelten Gebäudebestand pro Zelle aufsummiert.

$$
\frac{\sum_{\text {Gebiudezelle }} \mathrm{Q}_{\text {ERD }}}{\sum_{\text {Gebäudez Zelle }} \mathrm{Q}_{\mathrm{L}}^{*}}=\mathrm{L}^{1,2}
$$

Im Gegensatz zur Bestimmung des technischen Potenzials, welches auf der Festlegung einer Betriebsweise (fixierte Umsatzrate und Jahresarbeitsstunden der Erdwärmeanlage) basiert, muss die längenspezifische Jahresentzugsarbeit $\mathrm{Q}_{\mathrm{L}}^{*}[\mathrm{MWh} /(\mathrm{m} . \mathrm{a})]$ auf die verschiedenen Gebäudetypen und die damit verbundenen Betriebsweisen (Jahresarbeitsstunden) abgestimmt werden. Dies erfolgt unter Beibehaltung einer vorgegebenen Anlagengeometrie mittels Interpolation zwischen verschiedenen Betriebsweisen und den damit verbundenen Werten für $\mathrm{Q}_{\mathrm{L}}^{*}$. Gleichung (6) liefert in weiterer Folge einen zellenspezifischen Bedarf an Sondenmetern $\left(\mathrm{L}^{1}\right)$ bzw. Kollektorfläche $\left(\mathrm{L}^{2}\right)$. Um unrealistisch lange Sonden zu vermeiden, die in der Praxis kaum bis überhaupt nicht errichtet werden, kann eine maximale Sondentiefe $\left(\mathrm{L}_{\max }\right)$ definiert werden, die z.B. auf rechtliche Rahmenbedingungen (Meldepflicht bei der Montanbehörde) oder auf technischen Rahmenbedingungen fundiert. Im Rahmen der Studie GEO-Pot wurde eine maximale Sondentiefe von 110 Metern festgelegt, wobei es sich hierbei um einen in der Praxis üblichen Wert handelt. Die Anzahl der benötigten Tiefensonden zur Deckung des Heizwärmebedarfs ergibt sich aus dem Quotienten

$$
\mathrm{n}=\frac{\mathrm{L}^{1}}{\mathrm{~L}_{\max }}, \mathrm{n} \varepsilon \mathrm{R}
$$

Hierauf basierend kann unter Vorgabe des spezifischen Flächenbedarfs der Sonden (Querschnittsfläche, thermischer Einwirkungsbereich) der kumulierte Flächenbedarf der Erdwärmeanlage summiert werden. In einem abschließenden Bearbeitungsschritt wird das geothermische Deckungspotenzial unter Zellenbezug durch Quotientenbildung aus der verfügbaren Freifläche je Zelle und dem kumulierten Flächenbedarf der Erdwärmeanlage (Tiefensonde, Flachkollektor) berechnet.

\section{Vorläufige Schlussfolgerungen}

1. Vergleicht man die profilbezogenen Schätzung der Entzugsleistung [W/m] mittels VDI Richtlinie 4640 mit den Ergebnissen der numerischen Simulation nach Glück (2008), so lassen sich folgende Beobachtungen tätigen: (a) Unter Berücksichtigung vergleichbarer Sondengeometrien und Betriebskenndaten (Jahresarbeitsstunden) führt die Benutzung der VDI Richtlinie führt zu tendenziell erhöhten Entzugsleistungen, welche bei einer Seehöhe von $400 \mathrm{~m}$ ü.A. im Mittel um etwa $5 \mathrm{~W} / \mathrm{m}$ über jenen der numerischen Berechnung liegen, wobei Abweichungen bis zu $35 \mathrm{~W} / \mathrm{m}$ auftreten können (siehe auch Abb. 7). Unter Vorgabe einer Seehöhe von $1000 \mathrm{~m}$ ü.A. erhöhen sich zu beobachtenden mittleren Abweichungen sogar auf $19 \mathrm{~W} / \mathrm{m}$. (b) Die mittels VDI Richtlinie ermittelten profilspezifischen Entzugsleistungen fluktuieren weitaus stärker (Spannweite ca. $50 \mathrm{~W} / \mathrm{m}$ ) als jene der numerischen Simulation (Spannweite ca. $12 \mathrm{~W} / \mathrm{m}$ ). Die Ursachen der getätigten Beobachtungen liegen in der impliziten Berücksichtigung von fließendem Grundwasser in den VDI Schätzwerten, welche wiederum in der numerischen Simulation vernachlässigt wird. 


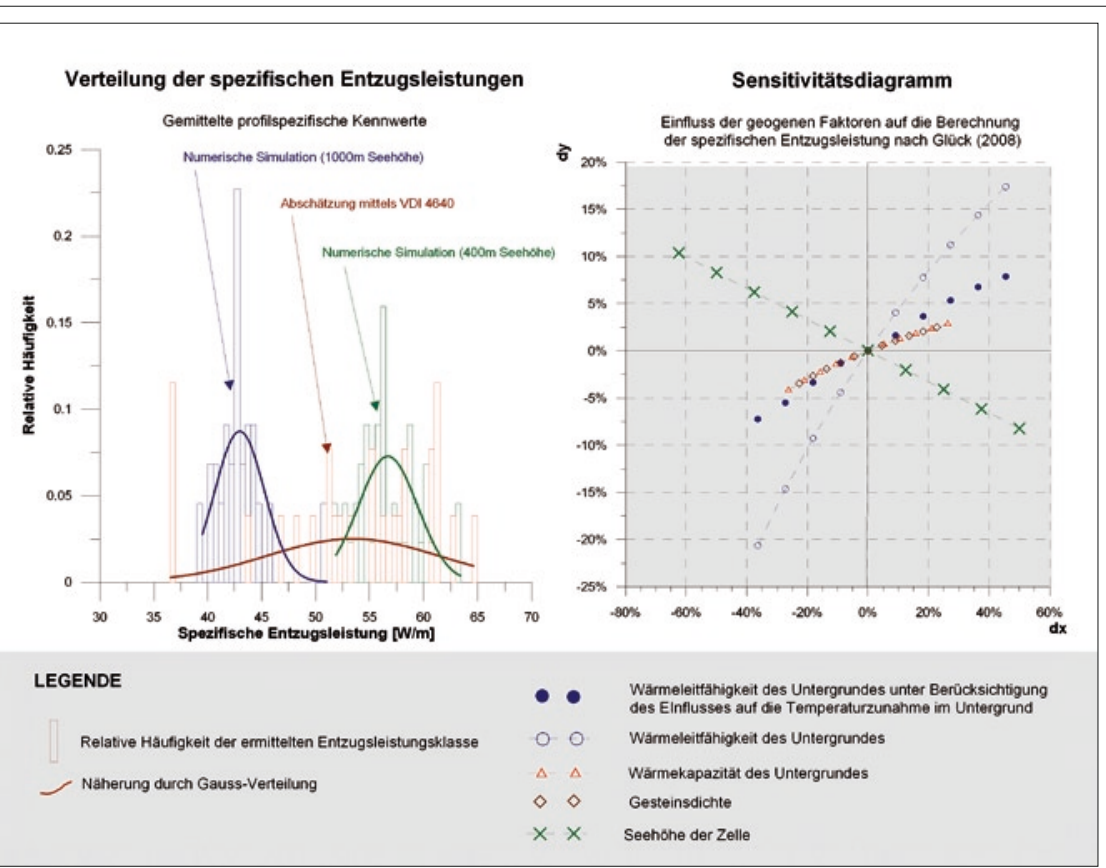

Abb. 7: Links - Relative Häufigkeitsverteilungen der ermittelten spezifischen Entzugsleistungen für die abgebildeten Referenzprofile. Rechts - Sensitivitätsdiagramm der geogenen Eingangsparameter zur Berechnung der thermischen Entzugsleistung nach Glück (2008). Ordinate - Änderung des Eingangswerts, Abszisse - Änderung der Entzugsleistung.

Aus diesem Grund stellt die Berechnung der thermischen Entzugsleistungen nach Glück (2008) einen konservativen Ansatz zur Bestimmung des geothermischen Nutzungspotenzials dar. Da die Kennwerte der VDI Richtlinie aus empirischen Beobachtungen an bestehenden Anlagen kompiliert werden, die vermutlich in einem begrenzten Höhenintervall (vorrangig Sonden aus Beckenregionen Deutschlands) erhoben worden sind, versagt die Richtlinie bei größeren Lagehöhen. Neben dem fehlenden Einfluss von fließendem Grundwasser lassen sich die deutlich eingegrenzten Wertebereiche der mittels numerischer Simulation nach Glück (2008) ermittelten mittleren profilspezifischen Entzugsleistungen durch folgenden Rückkoppelungseffekt zwischen Wärmeleitfähigkeit und Temperaturverteilung im Untergrund erklären (siehe auch Abb. 7, rechts). Bei steigender Wärmeleitfähigkeit des Untergrunds reduziert sich jedoch bei Vorgabe eines konstanten terrestrischen Wärmeflusses die Temperaturzunahme mit steigender

\section{LITERATUR}

Biermayr P et al. (2005) Maßnahmen zur Minimierung von Reboundeffekten bei der Sanierung von Wohngebäuden (MARESI), Berichte aus Energie- und Umweltforschung, Bundesministerium für Verkehr, Innovation und Technologie, rium

Frey, K et al. (1994) Handbuch für Energieberater, Joanneum Research, Institut für Energieforschung, Graz

Glück B (2008) Simulationsmodell "Erdwärmesonden zur wärmetechnischen Beurteilung von Wärmequellen, Wärmesenken und Wärme-/ Kältespeichern, Rud. Otto Meyer-Umwelt-Stiftung Hamburg

Glück B (2009) Simulationsmodell „Erdwärmekollektor" zur wärmetechnischen Beurteilung von Wärmequellen, Wärmesenken und Wärme- / Kältespeichern, Rud. Otto Meyer-Umwelt-Stiftung Hamburg

Marti J (2009) Simulation von Wärmepumpensystemen in Polysun, Vela Solaris AG, Winterthur, 15. Wärmepumpentagung, 24. Juni 2009, Burgdorf, Schweiz

OIB Richtlinie 6 (2007) Leitfaden Energietechnisches Verhalten von Gebäuden Version 2.6, Ös-
Tiefe (geothermischer Gradient). Die Überlagerung dieser entgegenwirkenden Phänomene führt zu einer deutlich reduzierten Abhängigkeit der thermischen Entzugsleistung von der Wärmeleitfähigkeit.

2. Aufgrund der unter Punkt 1 angeführten Aussagen hat sich gezeigt, dass die Sensitivität oberflächennaher geothermischer Potenziale gegenüber den technischen Bodenmerkmalen wesentlich geringer ausgeprägt ist als gegenüber den bestehenden Freiflächenanteilen. Aufgrund der Unsicherheiten in der Abschätzung der zellenbezogenen Freiflächenanteile ist die Vorgabe an die Komplexität der Berechnung der spezifischen thermischen Entzugsleistungen in Abhängigkeit geogener Faktoren beschränkt. Aus diesem Grund wurde bei der Bestimmung der technischen Entzugsleistung auf komplexe Randbedingungen (z. B. Schwankungen der Bodentemperatur in oberflächennahen Bodenschichten) verzichtet.

3. Die derzeitige restriktive Datenschutzpolitik erschwert eine kleinräumige Abbildung und Modellierung von gebäudebezogenen Energiefragen in hohem Maße. In Kombination mit der wenig kompakten Siedlungsstruktur in Österreich werden somit zukunftsweisende und möglichst aktuelle Aussagen über die räumlich differenzierte Nachfrage nach Energie für den Gebäudebestand und ihre Einflussfaktoren nur für dichter besiedelten Gebiete ermöglicht, während für die weit verbreiteten klassischen Einfamilienhausbereiche (die speziell für geothermische Nutzung grundsätzlich interessant sind) nur sehr allgemeine Aussagen möglich sind, da keine Daten zu Zellen mit weniger als 4 Gebäuden zur Verfügung gestellt werden. Eine Lösung ähnlich dem Schweizer Modell ${ }^{3}$ wäre erstrebenswert.

\section{Danksagung}

Die Projektgruppe möchte den verschiedenen Ämtern der Österreichischen Landesregierungen Dank für die zur Verfügung gestellten wasserwirtschaftlichen und anlagentechnischen Kenndaten aussprechen. Weiterer Dank gilt der Pla-

Schubert G (red.) (2003) Die Hydrogeologisch Karte von Österreich 1:500.000, Geologische Bundesanstalt Wien

Statistik Austria (2008) Statistisches Jahrbuch Österreichs 2008, Bundesanstalt Statistik Österreich, Wien

VDI 4640 (2001) Thermische Nutzung des Untergrunds, Erdgekoppelte Wärmepumpenanlagen, Blatt 1 (Entwurf), VDI e.V., Düsseldorf
Für die Analyse und Modellierung werden sämtliche Daten der Forschung zur Verfügung gestellt, sofern bei der Veröffentlichung der aggregierten Ergebnisse der Datenschutz wieder gewährleistet ist 
nungsgemeinschaft Ost (PGO) für die Vermittlung wichtiger Kontakte zur Evaluierung der Freiflächenanteile. Des Weiteren sei den Mitarbeiterinnen der Geologischen Bundesanstalt, Fr. Brüstle sowie Fr. Zekiri für die Durchführung der numerischen Simulationen zur Berechnung der spezifischen Entzugsleistungen gedankt. Herrn HR Dr. Walter Kollmann (Geologische Bundesanstalt Wien) sei abschließend für die Zurverfügungstellung von Bodentemperaturdaten aus eigenen Untersuchungen in den Tiefen 1.5 und 3 Meter unter GOK gedankt, die aus Platzgründen in der vorliegenden Arbeit nicht explizit beschrieben werden können.
Korrespondenz:

Mag. Gregor Götz

Geologische Bundesanstalt Wien

E-Mail: gregor.goetz।@geologie.ac.at

DI Viktoria Ostermann

Technische Universität Wien

Institut für Geotechnik Forschungsbereich für Grundbau und Bodenmechanik

DI Robert Kalasek

Technische Universität Wien

Institut für Stadt- und Regionalforschung

Dr. Richard Heimrath

Technische Universität Graz

Institut für Wärmetechnik

DI Peter Steckler

Technische Universität Wien

Institut für Verkehrswissenschaften

Forschungsbereich für Eisenbahnwesen

Verkehrswirtschaft und Seilbahnen
DI (FH) Andreas Zott

Austrian Institute of Technology

Dr. Andreas Novak

Universität Wien

Lehrstuhl für Industrie, Energie und Umwelt

Mag. Gudrun Haindlmaier

Technische Universität Wien

Institut für Stadt- und Regionalforschung

DI Robert Hackl

Technische Universität Wien

Institut für Stadt- und Regionalforschung

DI Siavaush Shadlau

Geologische Bundesanstalt Wien

Heinz Reitner

Geologische Bundesanstalt Wien 